\title{
Molecular mechanisms of "off-on switch" of activities of human IDH1 by tumor-associated mutation R132H
}

\author{
Bei Yang ${ }^{1,2}$, Chen Zhong ${ }^{1}$, Yingjie Peng ${ }^{1,2}$, Zheng Lai ${ }^{1}$, Jianping Ding ${ }^{1}$ \\ ${ }^{I}$ State Key Laboratory of Molecular Biology and Research Center for Structural Biology, Institute of Biochemistry and Cell Biol- \\ ogy, Shanghai Institutes for Biological Sciences, Chinese Academy of Sciences, Shanghai 200031, China; ${ }^{2}$ Graduate School of \\ Chinese Academy of Sciences, 320 Yueyang Road, Shanghai 200031, China
}

Human cytosolic NADP-IDH (IDH1) has recently been found to be involved in tumorigenesis. Notably, the tumorderived IDH1 mutations identified so far mainly occur at Arg132, and mutation R132H is the most prevalent one. This mutation impairs the oxidative IDH activity of the enzyme, but renders a new reduction function of converting $\alpha$-ketoglutarate $(\alpha \mathrm{KG})$ to 2-hydroxyglutarate. Here, we report the structures of the R132H mutant IDH1 with and without isocitrate (ICT) bound. The structural data together with mutagenesis and biochemical data reveal a previously undefined initial ICT-binding state and demonstrate that IDH activity requires a conformational change to a closed pre-transition state. Arg132 plays multiple functional roles in the catalytic reaction; in particular, the R132H mutation hinders the conformational changes from the initial ICT-binding state to the pre-transition state, leading to the impairment of the IDH activity. Our results describe for the first time that there is an intermediate conformation that corresponds to an initial ICT-binding state and that the R132H mutation can trap the enzyme in this conformation, therefore shedding light on the molecular mechanism of the "off switch" of the potentially tumor-suppressive IDH activity. Furthermore, we proved the necessity of Tyr139 for the gained $\alpha$ KG reduction activity and propose that Tyr139 may play a vital role by compensating the increased negative charge on the C2 atom of $\alpha \mathrm{KG}$ during the transfer of a hydride anion from NADPH to $\alpha$ KG, which provides new insights into the mechanism of the "on switch" of the hypothetically oncogenic reduction activity of IDH1 by this mutation.

Keywords: isocitrate dehydrogenase; NADP; catalytic mechanism; crystal structure; cancer metabolism Cell Research (2010) 20:1188-1200. doi:10.1038/cr.2010.145; published online 26 October 2010

\section{Introduction}

Isocitrate dehydrogenases (IDHs) are a family of enzymes that catalyze the oxidative decarboxylation of isocitrate (ICT) into $\alpha$-ketoglutarate $(\alpha \mathrm{KG})$ using either NADP or NAD as a cofactor. Human cells express three distinct IDH enzymes: cytosolic NADP-dependent IDH1, mitochondrial NADP-dependent IDH2, and

Correspondence: Jianping Ding

Tel: +86-21-5492-1619; Fax: +86-21-5492-1116

E-mail: jpding@sibs.ac.cn

Abbreviations: ICT (isocitrate); $\alpha \mathrm{KG}$ ( $\alpha$-ketoglutarate); 2HG (2-hydroxyglutarate); IDH (isocitrate dehydrogenase); NADP-IDH (NADP-dependent IDH); EcIDH (E. coli NADP-IDH); IDH1 (human cytosolic NADP-IDH); PmIDH (porcine mitochondrial NADP-IDH); Idp1 (S. cerevesiae mitochondrial NADP-IDH); GBM (glioblastoma multiforme)

Received 29 June 2010; revised 16 August 2010; accepted 1 September 2010; published online 26 October 2010 mitochondrial NAD-dependent IDH3. Recently, mutations in IDH1 have been correlated with certain brain tumors, with the prevalence of 54\%-94\% in grade II-III astrocytomas, oligodendrogliomas, and oligoastrocytomas [1-6], 50\%-88\% in secondary glioblastoma multiforme (GBM) [1-7], and 33\%-50\% in adult primitive neuroectodermal tumor $[1,7]$. GBM is the most aggressive brain tumor with almost invariably fatal prognosis, and the association of IDH1 mutation with the proneural subtype of GBM has important implications for future diagnosis and therapies for patients with GBM [8]. Besides brain tumors, $I D H 1$ mutations have also been detected in other cancers including acute myeloid leukemia [9], B-acute lymphoblastic leukemia [10], colorectal cancer [11], and prostate cancer [10], with low frequencies. The closely related IDH2 has also been documented to be mutated in several types of tumors albeit with low frequencies (1\%-7\%) $[3,6]$. 
It is striking to note that all the IDH mutations identified so far occur at Arg132 of IDH1 or the equivalent residue Arg172 of IDH2 heterozygously [1-7]. Among the identified IDH1 mutations, R132H is the most prevalent one in gliomas [1-6] constituting up to $92.7 \%$ of the total mutations, while $\mathrm{R} 132 \mathrm{C}$ and $\mathrm{R} 132 \mathrm{~S}$ were found with a percentage of $3.6 \%$ and $1.8 \%$, respectively [1]. Recently, two mechanisms by which the Arg132 mutations contribute to tumorigenesis have been proposed $[12,13]$. One hypothesis is that IDH1 may function as a tumor suppressor, and the impairment of the IDH activity by the Arg 132 mutations plays a tumorigenic role in part through activation of the HIF-1 pathway due to decreased $\alpha K G$ production [12]. The other is that the $\mathrm{R} 132 \mathrm{H}$ mutant gains a new activity of catalyzing NA$\mathrm{DPH}$-dependent reduction of $\alpha \mathrm{KG}$ to 2-hydroxyglutarate (2HG), which plays a role in the development and progression of malignant brain tumors [13]. The two possibilities are not mutually exclusive, and hence the R132 mutation might contribute to the tumorigenesis process by both "off switch" of the ICT dehydrogenase activity and "on switch" of the $\alpha \mathrm{KG}$ reduction activity. The molecular mechanisms underlying the "off-on switch" of the activities, however, are still unclear.

Previously, we have determined the crystal structures of the wild-type dimeric IDH1 $\left(\mathrm{IDH} 1^{\mathrm{R} / \mathrm{R}}\right)$ in complexes with NADP and with NADP, ICT, and $\mathrm{Ca}^{2+}$, and showed that the assembly of the active site is a complicated process involving concerted inter-domain motion and conformational changes of two subunits in a cooperative manner and that the enzyme may employ a self-regulatory mechanism to regulate its activity [14]. In the IDH1 $1^{\mathrm{R} / \mathrm{R}}$ NADP-ICT-Ca quaternary complex, Arg132, which is strictly conserved in all NADP-dependent IDHs (NADPIDHs) [15], interacts with ICT [14], providing an explanation for the substantially decreased $K_{\mathrm{m}}$ (ICT) values of the tumor-derived mutants [12]. Besides the decreased $K_{\mathrm{m}}$, these mutants also show decreased $V_{\max }[12]$ or $k_{\text {cat }}[13]$ for ICT dehydrogenation and more surprisingly acquire a new function of $\alpha \mathrm{KG}$ reduction [13], leading us to speculate that Arg132 may play other functional roles in addition to ICT binding in the catalysis. To further dissect the functional role(s) of Arg132 in the catalytic reaction and to explore the mechanistic role of the $\mathrm{R} 132 \mathrm{H}$ mutation in tumorigenesis, we carried out structural and functional analyses of the R132H mutant IDH1. Given that the maternally and paternally derived copies of each gene on autosomes are simultaneously expressed at comparable levels [16], the heterozygous R132H allele of $I D H 1$ may lead to three forms of IDH1 proteins in vivo, namely $\mathrm{IDH} 1^{\mathrm{R} / \mathrm{R}}$, heterodimeric mutant IDH1 $\left(\mathrm{IDH} 1^{\mathrm{R} / \mathrm{H}}\right)$, and homodimeric mutant IDH1 $\left(\mathrm{IDH} 1^{\mathrm{H} / \mathrm{H}}\right)$. We report here the crystal structures of IDH $1^{\mathrm{H} / \mathrm{H}}$ in complexes with NADP and with NADP and ICT, and IDH $1^{\mathrm{R} / \mathrm{H}}$ in complex with NADP and ICT, respectively. Furthermore, mutagenesis, kinetic, and thermodynamic studies were performed to explore the catalytic mechanisms of the ICT dehydrogenation reaction and the $\alpha \mathrm{KG}$ reduction reaction. The structural and biochemical data together not only reveal the molecular basis for the impairment of the IDH activity by the tumor-associated $\mathrm{R} 132 \mathrm{H}$ mutation, but also provide insights into the molecular mechanism underlying the gained $\alpha \mathrm{KG}$ reduction activity of the mutant IDH1.

\section{Results and Discussion}

\section{Overall structures of the R132H mutant complexes}

The structures of IDH $1^{\mathrm{H} / \mathrm{H}}$ in complexes with NADP and with NADP and ICT were determined at $3.4 \AA$ and $2.8 \AA$ resolutions, respectively. The structure of $\mathrm{IDH} 1^{\mathrm{R} / \mathrm{H}}$ in complex with NADP and ICT was determined at $3.2 \AA$ resolution. All the three structures belong to space group $P_{3} 2_{1} 2$ with similar cell parameters and contain two IDH1 subunits forming a dimer in the asymmetric unit (Table 1). Each IDH1 subunit consists of three domains: a large domain (residues 1-103 and 286-414), a small domain (residues 104-136 and 186-285), and a clasp domain (residues 137-185; Figure 1A). In the structure of the IDH $1^{\mathrm{H} / \mathrm{H}}$-NADP complex, there is an NADP bound at the NADP-binding site of each subunit even though no NADP was added in the crystallization solution, indicating that similar to the wild-type IDH1 [14], the mutant IDH1 was co-purified with a tightly bound NADP from the expression system. In the structures of both IDH $1^{\mathrm{H} / \mathrm{H}}$ NADP-ICT and IDH $1{ }^{\mathrm{R} / \mathrm{H}}$-NADP-ICT complexes, in each subunit, there is an NADP bound at the NADP-binding site and an ICT bound at a site composed of NADP and residues Thr77, Ser94, and Arg100 of the large domain (Figure 1A and 1B) which is distinct from the ICTbinding site in the IDH $1^{\mathrm{R} / \mathrm{R}}$-NADP-ICT-Ca complex [14] (see discussion later). In all the mutant IDH1 complexes, two key structural elements encompassing residues 132141 and residues 271-286 (designated as seg1 and seg2, respectively) exhibit great flexibility with the majority of them being disordered (Supplementary information, Table S1; see discussion later). In the IDH $1^{\mathrm{R} / \mathrm{H}}$-NADPICT complex, residue 132 cannot be distinguished as Arg or His, which may be due to the medium resolution of the structure or reflect a random distribution of the wildtype and mutant IDH1 in both subunits.

The R132H mutant complexes assume asymmetric open conformations

Previously, we have shown that in the IDH $1{ }^{\mathrm{R} / \mathrm{R}}$-NADP 
Table 1 X-ray diffraction data and structure refinement statistics

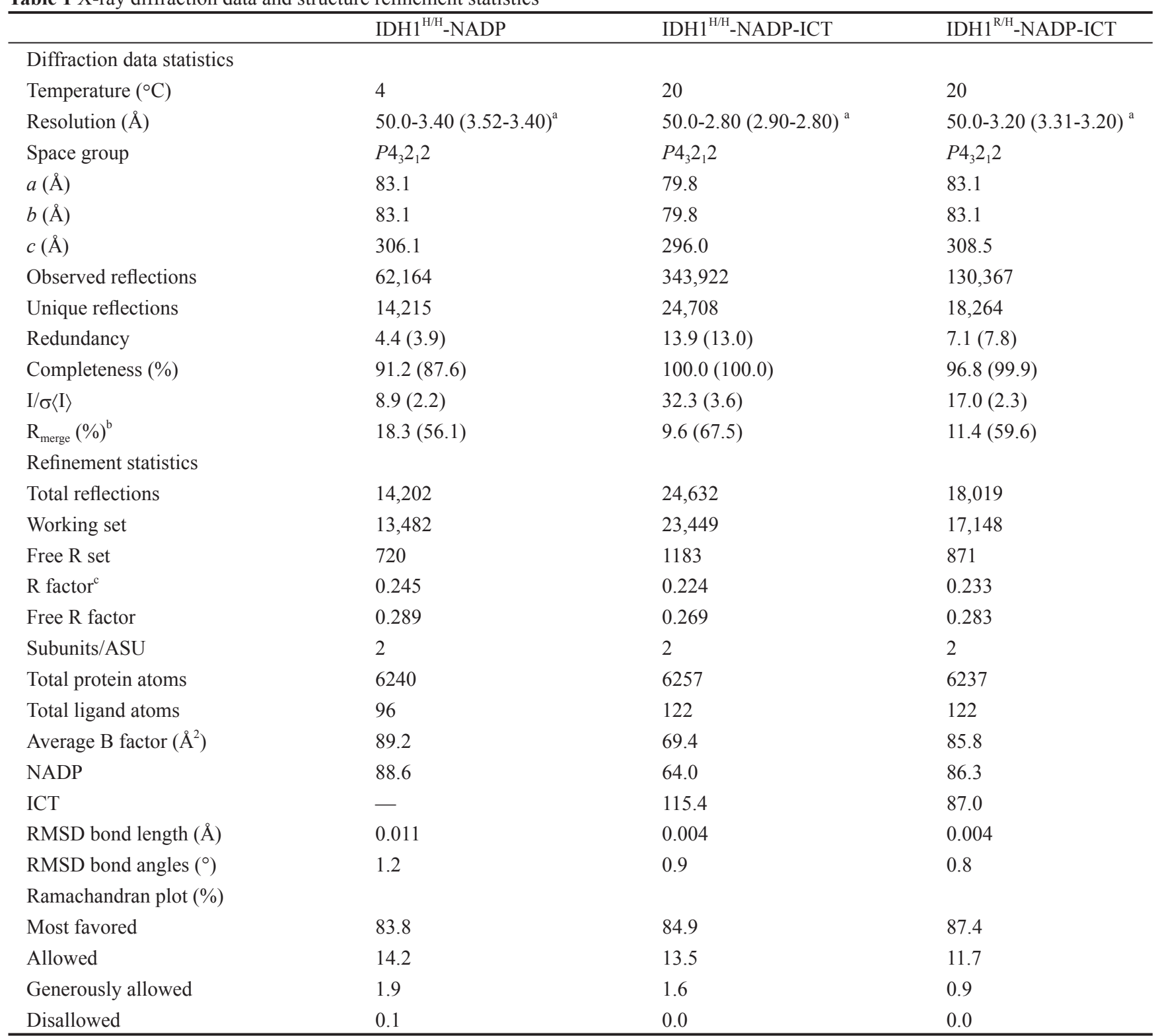

${ }^{\mathrm{a}}$ The numbers in parentheses refer to the highest resolution shell.

${ }^{\mathrm{b}} \mathrm{R}_{\text {merge }}=\Sigma_{h k l} \Sigma\left|\mathrm{I}_{i}(h k l)_{i}-\langle\mathrm{I}(h k l)\rangle\right| \Sigma_{h k l} \Sigma_{i} \mathrm{I}_{i}(h k l)$

${ }^{\mathrm{c}} \mathrm{R}$ factor $=\Sigma|| \mathrm{F}_{\mathrm{o}}|-| \mathrm{F}_{\mathrm{c}}|/ \Sigma| \mathrm{F}_{\mathrm{o}} \mid$.

complex, the two subunits of the dimeric IDH1 adopt different conformations: subunit $\mathrm{A}$ assumes an open conformation, while subunit B adopts a quasi-open conformation [14]. Further binding of ICT induces substantial conformational changes of the large domain towards the small and clasp domains, leading to the formation of a closed conformation of both subunits in the IDH $1^{\mathrm{R} / \mathrm{R}}$-NADP-ICT-Ca complex [14]. Structural analysis of the three mutant IDH1 complexes shows that their overall structures are similar (with an RMSD of 0.5-
$1.1 \AA$ for $776 \mathrm{C} \alpha$ atoms), and intriguingly, the two subunits in each complex are asymmetric (with an RMSD of 1.2-1.7 $\AA$ for $388 \mathrm{C} \alpha$ atoms). Superposition of the corresponding subunits among the IDH1 structures shows that in all the mutant IDH1 complexes, subunit A takes an open conformation and subunit $\mathrm{B}$ a quasi-open conformation as in the IDH1 ${ }^{\mathrm{R} / \mathrm{R}}$-NADP complex (Figure 1C) rather than a closed conformation as in the IDH $1^{\mathrm{R} / \mathrm{R}}$-NADP-ICT-Ca complex (Figure 1D). Correspondingly, the width of the active site entrance (19.6-20.7 $\AA$ in subunit A and 17.5- 
A

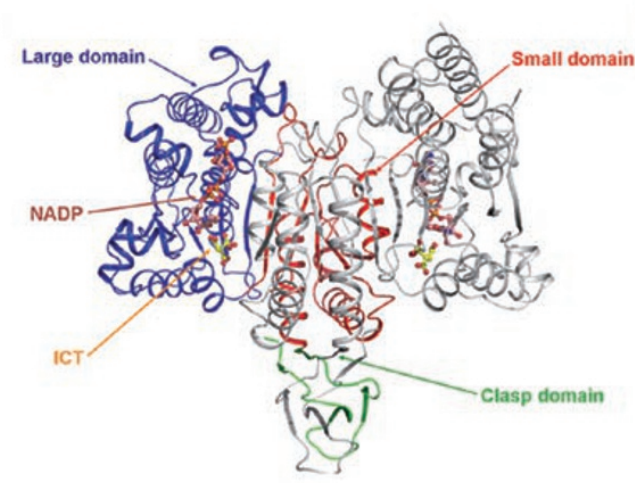

C

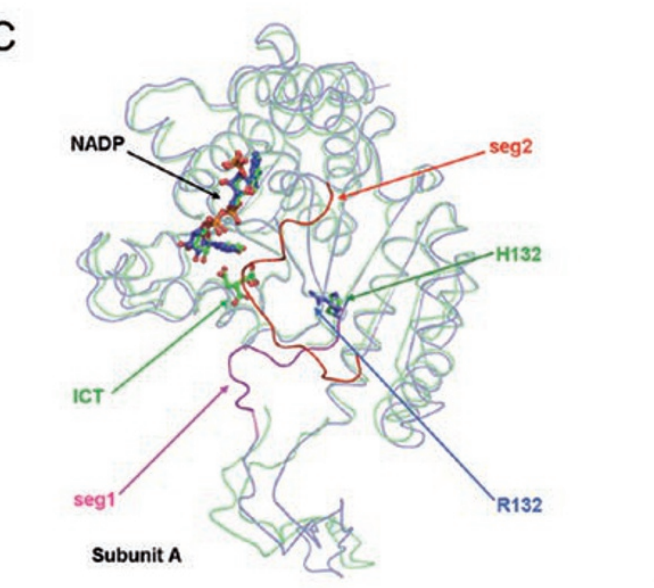

D

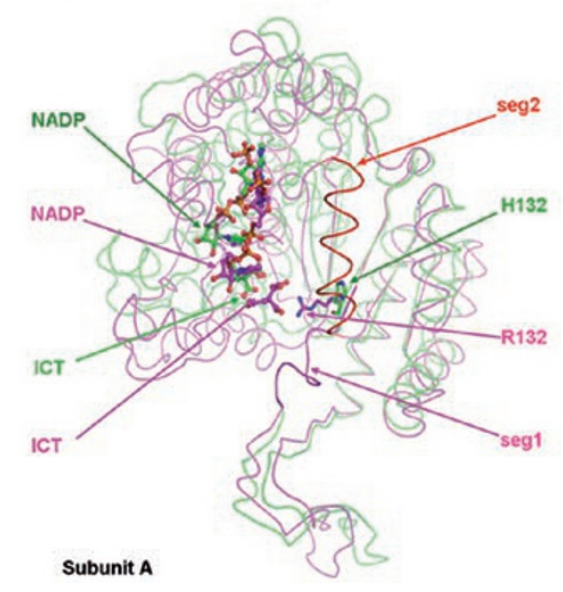

B

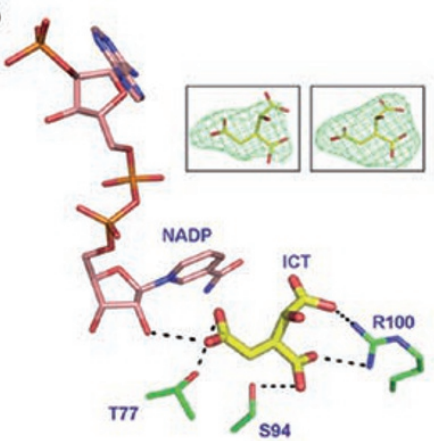

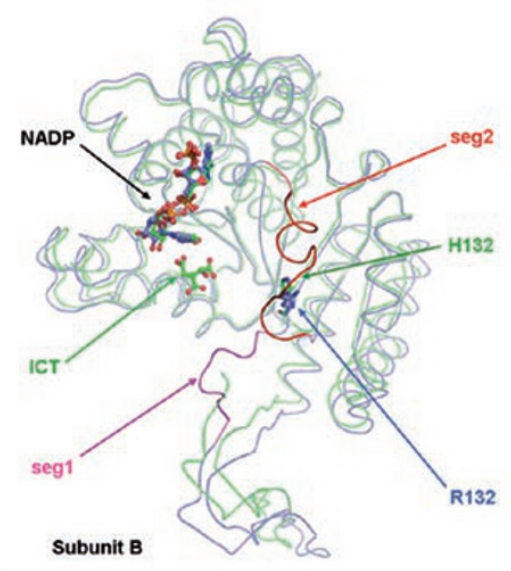

Subunit B

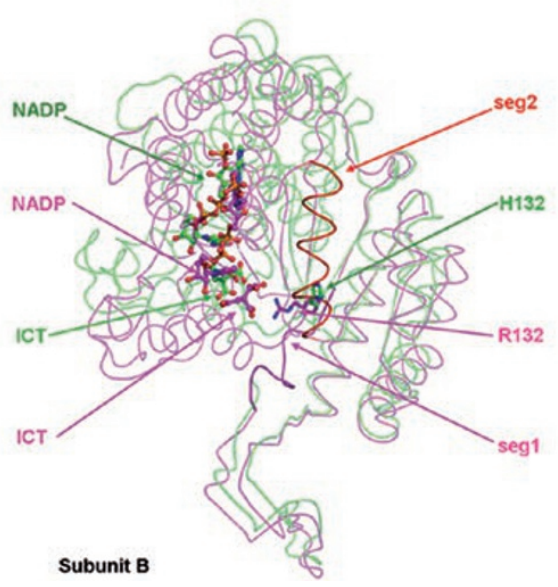

Figure 1 Structures of the R132H mutant IDH1 complexes. (A) Overall structure of the IDH1 ${ }^{\mathrm{H} / \mathrm{H}}-\mathrm{NADP}-\mathrm{ICT}$ complex. For simplicity, only one subunit of the IDH $1^{\mathrm{H} / \mathrm{H}}$-NADP-ICT complex is colored with the large domain in blue, the small domain in red, and the clasp domain in green, and the other subunit is colored in gray. The substrates are shown as ball-and-stick models with ICT in yellow and NADP in salmon. (B) Structure of the ICT-binding site in the IDH $1^{\mathrm{H} / \mathrm{H}}-\mathrm{NADP}-\mathrm{ICT}$ complex. The hydrogen-bonding interactions of ICT with the surrounding residues and NADP are indicated with dashed lines. The initial difference $F_{O}-F_{C}$ Fourier map (left) and the omit difference 2Fo-Fc Fourier map (right) for ICT are shown at $2.0 \sigma$ and $4.0 \sigma$ contour level, respectively. (C, D) Comparison of the respective subunits of the IDH1 $1^{H / H}-\mathrm{NADP}-\mathrm{ICT}$ complex with that of the $(\mathrm{C}) \mathrm{IDH} 1^{\mathrm{R} /}$ ${ }^{R}$-NADP and (D) IDH1 $1^{R / R}-N A D P-I C T-C a^{2+}$ complexes. Superposition of the structures was based on the small and clasp domains. The IDH $1^{\mathrm{H} / \mathrm{H}}$-NADP-ICT complex (green) assumes an open conformation in subunit $\mathrm{A}$ and a quasi-open conformation in subunit $\mathrm{B}$ similar to that of the IDH $1^{\mathrm{R} / \mathrm{R}}-\mathrm{NADP}$ complex (slate blue), rather than a closed conformation of the IDH $1^{\mathrm{R} / \mathrm{R}}-\mathrm{NADP}$ ICT-Ca ${ }^{2+}$ complex (magenta). In the mutant IDH1 complexes, the majority of seg1 and seg2 are disordered in both subunits; while in the wild-type IDH1 complexes, both segments have well-defined structures with different conformations (highlighted in magenta and red, respectively). His132 of the mutant and Arg132 of the wild-type IDH1, and the bound substrates are shown with ball-and-stick models. 
$18.8 \AA$ in subunit $\mathrm{B}$, defined as the distance between residue 76 of one subunit and residue 250 of the adjacent subunit) in the mutant IDH1 complexes is also comparable to that in the IDH1 ${ }^{\mathrm{RR}}$-NADP complex $(21.2 \AA$ and $18.7 \AA$, respectively). For simplicity, in the following discussion, we will use the structure of the IDH $1^{\mathrm{H} / \mathrm{H}}$-NADPICT complex as a representative of the mutant IDH1 complexes owing to its relatively higher resolution.

The $\mathrm{R} 132 \mathrm{H}$ mutation renders an increased flexibility of two key structural segments

It is intriguing to observe that two regions, seg1 and seg2, are largely disordered in all three mutant IDH1 complexes. In the wild-type IDH1 structures, seg1 forms a hinge region that dictates the conformational changes of the large domain induced by ICT binding, and seg2 forms a "regulatory segment" that undergoes substantial conformational change during the catalytic reaction and was proposed to regulate the enzymatic activity [14]. Specifically, seg1 adopts a coil structure in both subunits of the IDH $1^{\mathrm{R} / \mathrm{R}}$-NADP complex and a one-turn $\alpha$-helix in the IDH $1^{R / R}$-NADP-ICT-Ca complex similar to that ob- served in the other eukaryotic NADP-IDH structures [17, 18]. Seg2 takes a completely unwound loop structure in subunit A and a partially unwound one-turn $\alpha$-helix structure in subunit $\mathrm{B}$ in the IDH $1^{\mathrm{R} / \mathrm{R}}$-NADP complex, while it adopts a long $\alpha$-helix structure in the IDH1 $1^{\mathrm{R} / \mathrm{R}}$-NADPICT-Ca complex similar to that observed in all other NADP-IDH structures [17-21].

Detailed analyses of the wild-type IDH1 structures indicate that these two segments have extensive interactions with each other and Arg132 plays an important role in the interaction network (Figure $2 \mathrm{~A}$ and $2 \mathrm{~B}$, and Supplementary information, Table S2). In subunit A of the $I D H 1^{R / R}-N A D P$ complex, the $N \eta 1$ atom of $\operatorname{Arg} 132^{\mathrm{A}}$ (residues and structural elements of subunits $A$ and $B$ are denoted with superscripted letters A and B, respectively) imposes a salt bridge with the $\mathrm{O} \delta 2$ atom of $\operatorname{Asp} 275^{\mathrm{A}}(3.0$ $\AA$ ), while the $\mathrm{O}$ atom of Asp $275^{\mathrm{A}}$ forms a hydrogen bond with the $\mathrm{N}$ atom of $\operatorname{Tyr} 135^{\mathrm{A}}(2.9 \AA)$ and the $\mathrm{O}$ atom of Tyr $135^{\mathrm{A}}$ interacts with the $\mathrm{N}$ atom of $\operatorname{Gln} 277^{\mathrm{A}}(2.7 \AA$; Figure 2A left panel and Supplementary information, Table S2). In subunit B, the N 11 atom of $A r g 132^{B}$ forms a hydrogen bond with the $\mathrm{O} \delta 2$ atom of $A \operatorname{sn} 271^{\mathrm{B}}$ (3.3 $\AA$ )
A

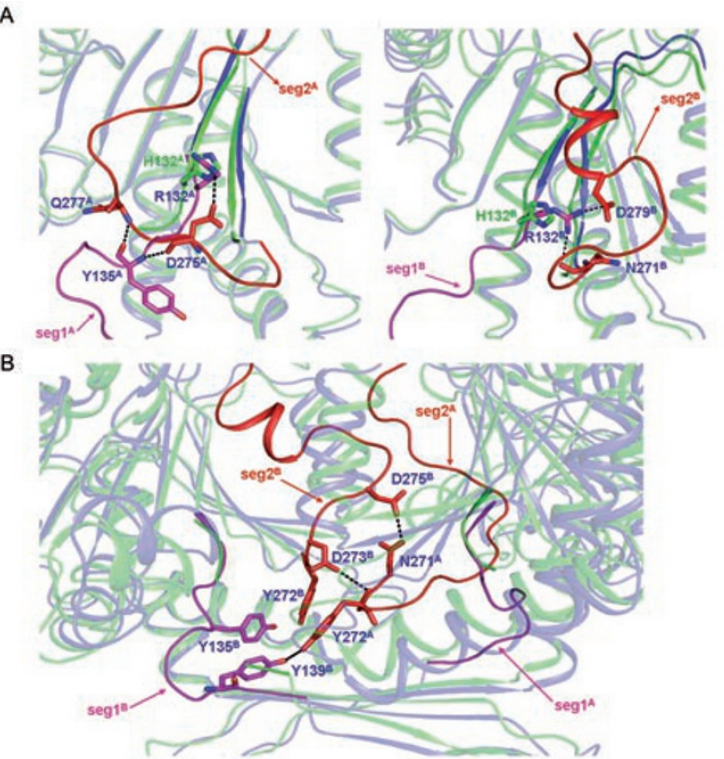

C

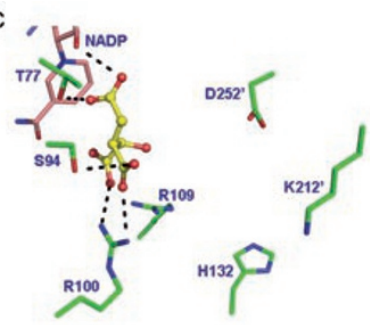

E

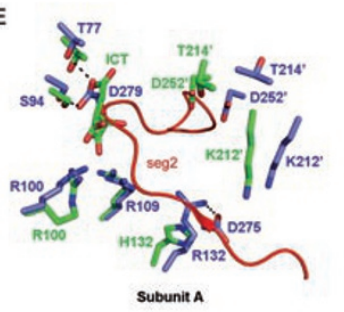

D
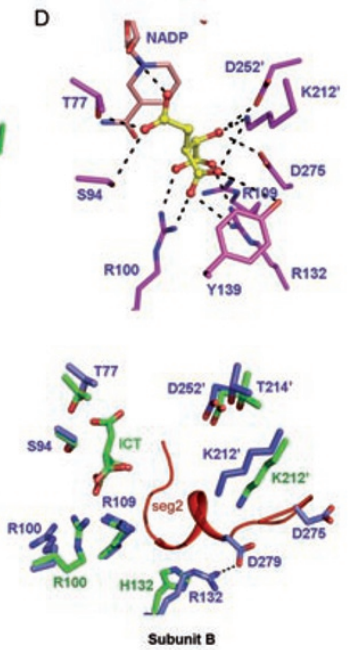

Figure 2 Comparison of the active sites between the R132H mutant and wild-type IDH1 complexes. (A) Comparison of the interactions between seg1 (red) and seg2 (magenta) in subunit A (left panel) and subunit B (right panel) of the IDH $1^{\mathrm{H} / \mathrm{H}}-\mathrm{NADP}$ ICT (green) and IDH1 $1^{\text {R/R }}$-NADP (slate blue) complexes. (B) Comparison of the interactions between seg2 of the two subunits in the IDH1 ${ }^{\mathrm{H} / \mathrm{H}}$-NADP-ICT (green) and IDH1 ${ }^{\mathrm{R} / \mathrm{R}}$-NADP (slate blue) complexes. The hydrophilic interactions are indicated with dashed lines and the involved residues are shown with stick models. (C) Structure of the ICT-binding site I in the IDH $1^{\mathrm{H} / \mathrm{H}}$ NADP-ICT complex. (D) Structure of the ICT-binding site II in the IDH $1^{\text {R/R }}$-NADP-ICT-Ca complex. Residues involved in ICT binding in the mutant IDH1 complex are shown with stick models in green and that in the wild-type IDH1 complex in magenta. Residues of the adjacent subunit are denoted with an apostrophe. ICT is shown with ball-and-stick model in yellow and NADP with stick model in salmon. (E) Comparison of the ICT-binding site I in subunit A (left panel) and subunit B (right panel) between the IDH1 $1^{R / R}$-NADP (slate blue) and IDH1 ${ }^{\mathrm{H} / \mathrm{H}}-\mathrm{NADP}-\mathrm{ICT}$ (green) complexes. Seg1 in the wild-type IDH1 complex is shown with red ribbons. 
and its $\mathrm{N} \eta 2$ atom forms a salt bridge with the $\mathrm{O} \delta 2$ atom of $\operatorname{Asp} 279^{\mathrm{B}}$ (3.1 A; Figure 2A right panel and Supplementary information, Table S2). At the dimer interface, hydrogen bonds form between the On atom of Tyr $139^{\mathrm{B}}$ and the $\mathrm{O} \eta$ atom of $\mathrm{Tyr} 272^{\mathrm{A}}(3.6 \AA)$, between the $\mathrm{N} \delta 2$ atom of $A s n 271^{\mathrm{A}}$ and the $\mathrm{O} \delta 2$ atom of $\mathrm{Asp} 275^{\mathrm{B}}(2.8 \AA)$, and between the $\mathrm{N}$ atom of Tyr2 $272^{\mathrm{A}}$ and the $\mathrm{O} \delta 2$ atom of Asp273 ${ }^{\mathrm{B}}$ (3.4 $\AA$; Figure 2B and Supplementary information, Table S2). Moreover, the aromatic rings of Tyr2 $272^{\mathrm{A}}$, Tyr $135^{\mathrm{B}}, \operatorname{Ty}_{13} 139^{\mathrm{B}}$, and Tyr $272^{\mathrm{B}}$ form a hydrophobic cluster, further strengthening the interactions of the two regions (Figure $2 \mathrm{~B}$ ). In the IDH $1^{\mathrm{R} / \mathrm{R}}-\mathrm{NADP}-\mathrm{ICT}-\mathrm{Ca}$ complex, the interactions between the two segments are even more intense (Supplementary information, Table $\mathrm{S} 2)$. The $\mathrm{N} \varepsilon$ atom of $\mathrm{Arg} 132^{\mathrm{A}}$ forms two hydrogen bonds with the $\mathrm{O}$ and $\mathrm{O} \delta 1$ atoms of $A \operatorname{sn} 271^{\mathrm{A}}(3.1 \AA$ and $2.9 \AA$, respectively) and its $\mathrm{N \eta} 2$ atom forms a hydrogen bond with the $\mathrm{O} \delta 1$ atom of $A s n 271^{\mathrm{A}}(2.9 \AA)$. In addition, the $\mathrm{O}$ atom of Gly $136^{\mathrm{A}}$ forms a hydrogen bond with the $\mathrm{N} \delta 2$ atom of $A s n 271^{\mathrm{A}}(3.3 \AA)$, and the On atom of Tyr $139^{\mathrm{A}}$ forms a hydrogen bond with the $\mathrm{O} \delta 1$ atom of $\operatorname{Asp} 275^{\mathrm{A}}(2.7$ $\AA)$. Corresponding interactions also exist among the residues of subunit B. At the dimer interface, the On atom of Tyr272 ${ }^{\mathrm{A}}$ forms two hydrogen bonds with the $\mathrm{O} \delta 1$ and $\mathrm{O} \delta 2$ atoms of $\mathrm{Asn} 273^{\mathrm{B}}$ (2.7 $\AA$ and $3.4 \AA$, respectively), and the $\mathrm{O} \delta 1$ atom of $A s p 275^{\mathrm{A}}$ forms a salt bridge with the $\mathrm{N} \zeta$ atom of Lys $212^{\mathrm{B}}(2.8 \AA)$, and vice versa. These results underscore the importance of Arg132 in the establishment of the interaction network and the stabilization of the two structural segments and thus, mutation of $\operatorname{Arg} 132$ to His would disrupt its interactions with the other residues and subsequently interactions between the two segments, leading to an increased flexibility of the two segments in the mutant IDH1 complexes.

It has been shown that the homodimeric IDH1 mutant has a significantly decreased IDH activity and intriguingly, the heterodimeric IDH1 mutant displays a comparable low IDH activity, suggesting that the two subunits of the dimeric IDH1 work in a cooperative manner [12]. Our structures strengthen this notion by showing that although ICT is bound with both subunits, the wild-type subunit of the heterodimer could not accomplish the conformational changes and concurrently, the two key structural segments exhibit remarkably increased flexibility. As in the wild-type enzyme, the conformational changes of the two segments and the inter-domain motion are induced by ICT binding, and a number of residues in these two segments are involved in ICT binding, metal ion coordination, and catalysis $[14,22]$; we speculate that the increased flexibility of these two regions rendered by the $\mathrm{R} 132 \mathrm{H}$ mutation might interfere with the ICT binding and/or the overall conformational changes of the enzyme, leading to the impairment of the IDH activity.

Table 2 Kinetic parameters for the IDH activity of the wild-type and homodimeric mutant IDH1 ${ }^{\mathrm{a}}$

\begin{tabular}{|c|c|c|c|c|c|}
\hline Enzyme & Specific activity & $K_{\mathrm{m}}(\mathrm{ICT})$ & $k_{\text {cat }}$ & $k_{\mathrm{cat}} / K_{\mathrm{m}}$ & $K_{\mathrm{D}}$ \\
\hline IDH1 & $\left(\mu \mathrm{mol} \mathrm{min} \mathrm{mg}^{-1} \mathrm{mg}^{-1}\right)$ & $(\mu \mathrm{M})$ & $\left(\mathrm{S}^{-1}\right)$ & $\left(\mathrm{S}^{-1} \mu \mathrm{M}^{-1}\right)$ & $(\mu \mathrm{M})$ \\
\hline Wild-type & $14.2 \pm 0.5$ & $6.39 \pm 0.55$ & $11.0 \pm 0.35$ & $1.72 \pm 0.16$ & $1.13 \pm 0.13$ \\
\hline S94A & $3.10 \pm 0.06$ & $60.7 \pm 5.5$ & $2.41 \pm 0.05$ & $0.040 \pm 0.00$ & \\
\hline $\mathrm{T} 77 \mathrm{~A} / \mathrm{S} 94 \mathrm{~A}$ & $0.52 \pm 0.01$ & $779 \pm 50$ & $0.40 \pm 0.01$ & $0.51 \pm 0.04 \times 10^{-3}$ & \\
\hline R109Q & $0.30 \pm 0.02$ & $9.63 \pm 1.13 \times 10^{3}$ & $0.23 \pm 0.02$ & $0.024 \pm 0.00 \times 10^{-3}$ & \\
\hline $\mathrm{R} 132 \mathrm{H}$ & $0.76 \pm 0.03$ & $1.28 \pm 0.13 \times 10^{3}$ & $0.60 \pm 0.02$ & $0.47 \pm 0.05 \times 10^{-3}$ & \\
\hline R132A & $2.63 \pm 0.22$ & $676 \pm 57$ & $2.05 \pm 0.17$ & $3.03 \pm 0.36 \times 10^{-3}$ & \\
\hline $\mathrm{R} 132 \mathrm{C}$ & $0.32 \pm 0.01$ & $1.17 \pm 0.06 \times 10^{3}$ & $0.25 \pm 0.01$ & $0.21 \pm 0.01 \times 10^{-3}$ & \\
\hline K212A & $0.013 \pm 0.000$ & $6.30 \pm 0.66$ & $0.011 \pm 0.000$ & $0.017 \pm 0.002$ & \\
\hline D252A & $0.032 \pm 0.001$ & $3.72 \pm 0.43$ & $0.024 \pm 0.001$ & $6.45 \pm 0.79 \times 10^{-3}$ & \\
\hline D275A & $1.62 \pm 0.06$ & $115 \pm 14$ & $1.27 \pm 0.05$ & $0.011 \pm 0.001$ & \\
\hline
\end{tabular}

${ }^{a}$ The specific IDH activities were measured under the standard assay conditions described in Materials and Methods. The $K_{\mathrm{m}}$ and $k_{\text {cat }}$ values were determined by varying the concentration of ICT while maintaining the concentrations of the other substrates at the standard saturating conditions. 
The R132H mutant complexes reveal a novel ICT-binding site

Comparison of the IDH $1^{\mathrm{H} / \mathrm{H}}$ complexes shows that in contrast to the wild-type IDH1, the ICT binding does not cause any significant domain movement in the mutant. In the IDH $1{ }^{\mathrm{H} / \mathrm{H}}$-NADP-ICT complex, although the two subunits of the homodimer display certain asymmetry, ICT binds at the same site which is far from the small domain and is distinct from the ICT-binding site in the IDH $1^{\mathrm{R} /}$ ${ }^{R}$-NADP-ICT-Ca complex (Figure 1D). Correspondingly, the interactions between ICT and the surrounding residues are different (Figure $2 \mathrm{C}$ and 2D). Specifically, the $\mathrm{O} 2$ atom of $\alpha$-carboxyl and $\mathrm{O} 5$ atom of $\beta$-carboxyl

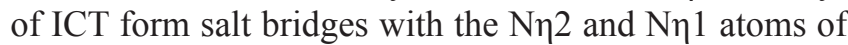
$\operatorname{Arg} 100$ (3.0 $\AA$ and $3.2 \AA$ ), respectively, and the O6 atom of $\beta$-carboxyl forms a hydrogen bond with the $\mathrm{O} \gamma$ atom of Ser94 (3.3 $\AA$ ). In addition, the $\gamma$-carboxyl forms two hydrogen bonds with the 2 '-OH group of NADP $(3.0 \AA)$ and the $\mathrm{O} \gamma 1$ atom of Thr77 (3.6 ̊; Figure 2C). Notably, in the mutant IDH1 complexes, the residues responsible for ICT binding (Thr77, Ser94, and Arg100) all belong to the large domain, and the respective interactions between ICT and the residues of the small domain in the wildtype IDH1 complexes are absent (Figure 2C and 2D). For convenience, we designate the ICT-binding site in the IDH $1{ }^{\mathrm{H} / \mathrm{H}}$-NADP-ICT complex as "site I" and that in the IDH $1{ }^{\mathrm{R} / \mathrm{R}}$-NADP-ICT-Ca complex as "site II".

So far, there is no structure of IDH1 in complex with ICT alone available. For porcine and yeast (Saccharomyces cerevesiae) mitochondrial NADP-IDHs (PmIDH and Idp1), the structures of the enzymes in complexes with ICT show that the binding of ICT, but not NADP, induces transformation of the enzyme from an open conformation to a quasi-closed conformation $[17,18]$. In these structures, ICT binds at a site close to site II and interacts with residues of both large and small domains in a similar way. Besides, seg 1 assumes an ordered structure containing a one-turn $\alpha$-helix and seg2 adopts a long $\alpha$-helix structure, similar to those observed in the $\mathrm{IDH} 1^{\mathrm{R} / \mathrm{R}}$-NADP-ICT-Ca complex [14]. These results suggest that for eukaryotic IDHs, the binding of ICT at site I appears to be associated with an open conformation of the enzyme with disordered structures of seg1 and seg2, while the binding of ICT at site II seems to be correlated to a closed conformation with ordered structures of the two segments.

\section{Kinetic studies of the wild-type and mutant IDH1}

To discern how the changes rendered by the $\mathrm{R} 132 \mathrm{H}$ mutation, including the increased flexibility of seg 1 and seg2 and the distinct binding mode of ICT, lead to impaired IDH activity, we carried out mutagenesis and biochemical studies towards Arg132 and other residues at the active site that have interactions with ICT and the metal ion. As shown in Table 2, the $V_{\max }$ and $K_{\mathrm{m}}$ (ICT) values of the wild-type IDH1 are comparable to that of PmIDH and E. coli NADP-IDH (EcIDH) [22-26]. Compared with the wild-type IDH1, the homodimeric R132H mutant manifests a 200-fold increase in the $K_{\mathrm{m}}$ whereas its specific activity is decreased to about $5 \%$; similarly, the $K_{\mathrm{m}}$ of the R132A mutant is also substantially increased by 106-fold while its specific activity is decreased to about $18 \%$ (Table 2). There is discrepancy in the IDH activity of the R132H mutant as Zhao et al. [12] showed a 2-fold decrease in the catalytic rate while Dang et al. [13] reported a 1000-fold reduction, and our data are in better agreement with that reported by Zhao et al. In addition, the kinetic parameters of the R132C, R132S, and R132L mutants are similar to that of the R132H mutant (Table 2). The Y139A and K212A mutants display < $1 \%$ activity (Table 2), indicating that Tyr139 and Lys212, like their counterparts in EcIDH and PmIDH [22, 25, 27], play critical roles in the catalytic reaction. Our data also show that mutations D252A and D275A have severe effects on the $K_{\mathrm{m}}$ and/or $k_{\text {cat }}$ due to their involvements in both ICT binding and catalysis (Table 2).

\section{Site I is an initial ICT-binding site}

As discussed above, in the IDH $1{ }^{\mathrm{H} / \mathrm{H}}$-NADP-ICT structure, ICT binds at site I and has interactions with residues Thr77, Ser94, and Arg100 of the large domain. Lys212 retains a position similar to that in the IDH1 ${ }^{\mathrm{R} / \mathrm{R}}$-NADPICT-Ca structure and thus is unable to interact with ICT (Figure 2C and 2D). In addition, Tyr139 of seg1 and Asp275 of seg2 appear to lose their interactions with ICT due to the increased flexibility of the two segments. As proper positioning of these key residues at the active site is critical for the enzymatic activity, the IDH $1{ }^{\mathrm{H} / \mathrm{H}}$-NADPICT structure is unlikely to represent a catalytically competent pre-transition state for the $\mathrm{R} 132 \mathrm{H}$ mutant that retains very low IDH activity (our data and [12]). Thus, we propose that formation of an active pre-transition state similar to the IDH $1^{\mathrm{R} / \mathrm{R}}$-NADP-ICT-Ca structure is a pre-requisite for the catalysis to take place. This leads us to the following questions: is site I an initial ICT-binding site and if yes, how does IDH1 transform from the initial ICT-binding state to the pre-transition state?

In the IDH $1{ }^{\mathrm{H} / \mathrm{H}}$-NADP complex, seg2 is disordered in both subunits and thus, site I is easily accessible and residues Thr77, Ser94, and Arg100 are available for ICT binding (Figure 2E). However, in the IDH $1^{\mathrm{R} / \mathrm{R}}$-NADP complex, seg2 is ordered but assumes different conformations in the two subunits, and site I is available only in subunit B, as in subunit A it is occupied by Asp279 ${ }^{\mathrm{A}}$ of 
seg2 which makes hydrogen bonds with both $T h r 77^{\mathrm{A}}$ and Ser94 ${ }^{\mathrm{A}}$ (Figure 2E). Among the three residues, Arg 100 is of particular interest as it is available for ICT binding in both subunits of the wild-type or mutant IDH1 binary complex and appears to play an important role in the interactions of the large domain with ICT due to its contribution of two salt bridges with ICT (Figure 2C and 2D). Our biochemical data show that the R100A mutation has drastic effects on both $K_{\mathrm{m}}\left(120\right.$-fold) and $k_{\text {cat }}(1 \%$; Table 2$)$, underscoring its pivotal roles in both ICT binding and catalysis. The T77A and S94A mutants possess about $26 \%$ and $22 \%$ activity, whereas their $K_{\mathrm{m}}$ values are increased by about 15- and 10-fold, respectively; and the double mutation shows a synergetic effect (Table 2), indicating that these two residues play more important roles in ICT binding than in catalysis. These results are consistent with the biochemical studies of the equivalent residues (Thr78, Ser95, and Arg101) of PmIDH [24, 26].

Considering that the $K_{\mathrm{m}}$ value can be affected by other factors such as the rate of conversion of the bound substrate to the product and hence may not accurately reflect the binding affinity between the substrate and the enzyme, we further analyzed the thermodynamics of ICT binding by the wild-type and mutant IDH1 using isothermal titration calorimetry (ITC; Figure 3). The ITC data show that the wild-type enzyme binds ICT with a

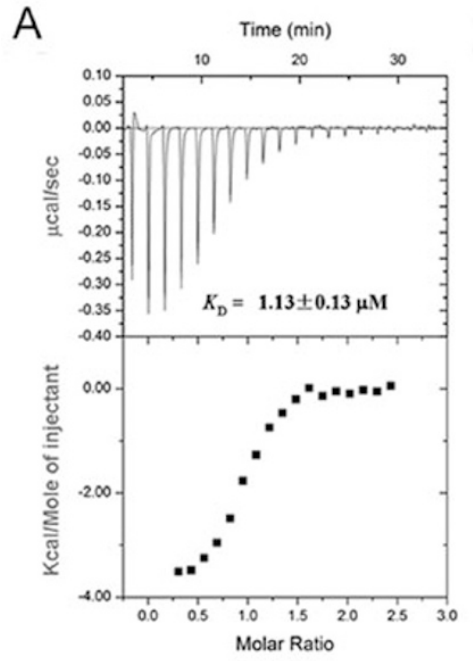

WT

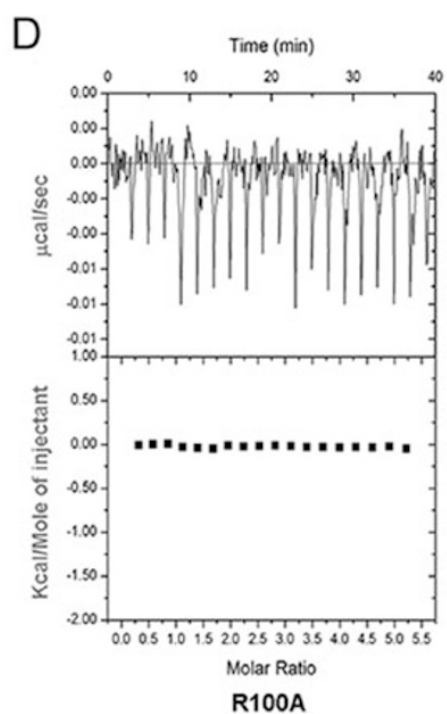

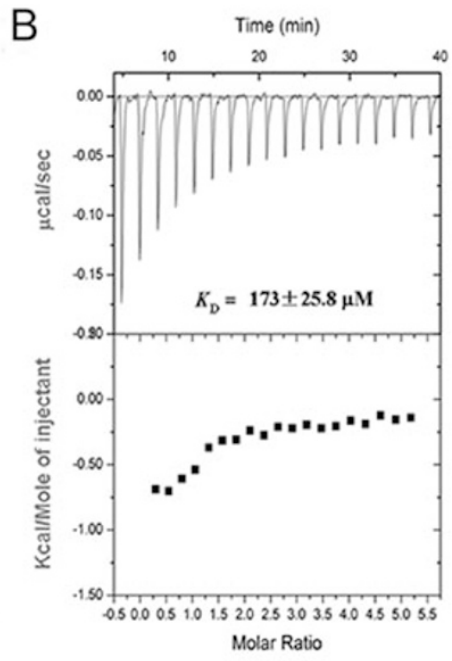

T77A

E

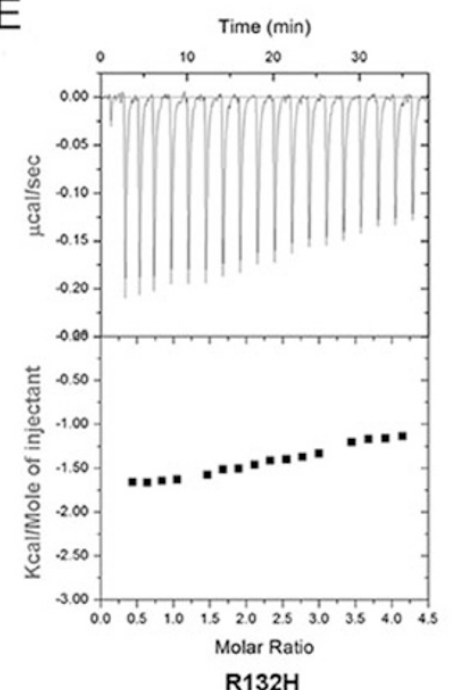

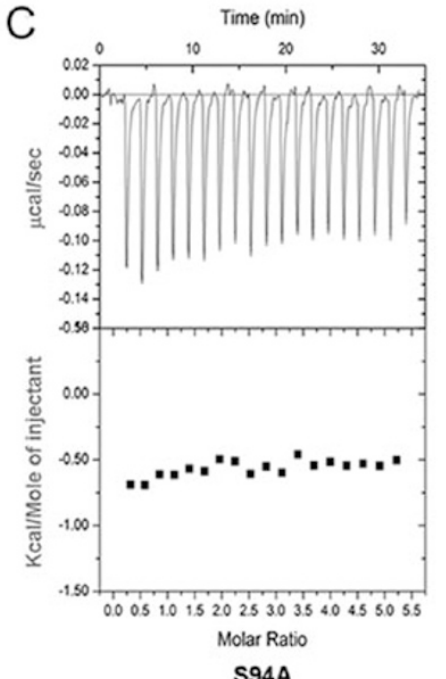

S94A

Figure 3 Thermodynamic characterization of the wild-type and mutant IDH1. (A-E) ITC data of ICT titration into the wild-type IDH1 and the T77A, S94A, R100A, and R132H mutants, respectively. For the R132H mutant, the concentration of ICT was doubled compared with that for the wild-type IDH1, and for the T77A, S94A, and R100A mutants, the concentrations of both ICT and protein were doubled for higher heat release. 
$K_{\mathrm{D}}$ value $(1.13 \mu \mathrm{M})($ Figure $3 \mathrm{~A}$ and Table 2$)$ comparable to the $K_{\mathrm{m}}$ value $(6.39 \mu \mathrm{M})$ as determined by the kinetics method (Table 2). In contrast, while the kinetic data show that the $K_{\mathrm{m}}$ values of the T77A and S94A mutants are only increased by 15 - and 10 -fold, respectively (Table 2 ), the ITC data show that the $K_{\mathrm{D}}$ value of the T77A mutant is increased by 153 -fold and the S94A mutant shows a distinct profile with no measurable $K_{\mathrm{D}}$ value (Figure $3 \mathrm{~B}$ and $3 \mathrm{C}$, and Table 2), suggesting that Thr77 and Ser94 might not be very critical in the ICT binding in the pretransition state but play important roles in the initial binding of ICT. Although the $K_{\mathrm{m}}$ values of the R100A and $\mathrm{R} 132 \mathrm{H}$ mutants are increased to the same order of magnitude (120- and 200-fold, respectively; Table 2), the two mutants have distinct thermodynamic profiles: the R132H mutant shows some binding ability with ICT, whereas the R100A mutant has no detectable binding (Figure 3D and 3E), indicating that mutation of Arg 100 has much greater impact on the $K_{\mathrm{D}}$ compared with mutation of Arg132. These results together indicate that site I is an initial ICT-binding site and residues Thr77, Ser94, and Arg100 of the large domain are responsible for the binding of ICT at site I.

After the initial binding of ICT, the enzyme needs to convert from the open conformation to the closed conformation with the corresponding movement of ICT from site I to site II to reach the pre-transition state. A detailed analysis of the IDH $1{ }^{\mathrm{H} / \mathrm{H}}$-NADP-ICT structure shows that the $\mathrm{N \eta} 1$ atom of $\operatorname{Arg} 109$ may interact with the $\alpha$-carboxyl of ICT (4.6 $\AA$ and $4.9 \AA$ from the $\alpha$-carboxyl oxygen atoms, respectively), which may trigger such conformational changes. As the IDH $1{ }^{\mathrm{H} / \mathrm{H}}$-NADP-ICT complex assumes a conformation similar to that of the IDH $1^{\mathrm{R} / \mathrm{R}}$ NADP complex, Arg109 may exert such a function in the wild-type enzyme. It is also possible that Arg132 may play a similar role in the wild-type enzyme. Analysis of the IDH $1^{\mathrm{R} / \mathrm{R}}$-NADP complex shows that Arg132 forms salt bridges with Asp275 in subunit A and Asp279 in subunit B. At these positions, Arg 132 would be too far to interact with ICT at site I. However, the ICT binding to site I might lead to disruption of the interactions between Arg132 and seg2 and thus, the "freed" Arg132 might be able to extend its side chain to interact with ICT and therefore initiate and/or participate in the conformational changes. The notion that Arg109 and Arg132 function in triggering the conformational changes is supported by the biochemical data that mutations of both Arg109 and $\operatorname{Arg} 132$ cause drastic increase in the $K_{m}$ (Table 2).

Mechanism of the impaired IDH activity of the $\mathrm{R} 132 \mathrm{H}$ mutant

As discussed above, the IDH $1{ }^{\mathrm{H} / \mathrm{H}}$-NADP-ICT structure unexpectedly captures a snapshot representing a transient state of the ICT dehydrogenation reaction in which ICT is initially bound and the active site is partially assembled (Figure 4A). For the complete assembly of the active site and for the catalysis to take place, the enzyme has to convert from the open conformation of the initial ICT-binding state to the closed conformation of the pretransition state represented by the IDH $1^{\mathrm{R} / \mathrm{R}}$-NADP-ICTCa structure, accompanied by the movement of ICT from site I to site II (Figure 4A). During the catalytic reaction, Arg132 exerts multiple functional roles via direct interactions with ICT, facilitation of the conformational changes, and formation of the active site (this study and the study by Xu et al. [14]). Mutation of Arg132 to His does not prohibit the binding of ICT, instead it constrains the enzyme in the inactive, initial ICT-binding state and prevents it from undergoing the conformational changes required to form the active, pre-transition state (Figure $4 \mathrm{~A})$.

Intriguingly, our results also show that the structure of the IDH $1^{\mathrm{R} / \mathrm{H}}$-NADP-ICT complex resembles the corresponding structure of the homodimeric $\mathrm{IDH}^{\mathrm{H} / \mathrm{H}}$ rather than the wild-type enzyme, supporting the notion that trap of the mutant subunit in the initial ICT-binding state has a negative impact on the wild-type subunit [12] due to the cooperation of the two subunits in ICT binding and conformational changes via the two key structural segments (seg1 and seg2) [14]. Our findings indicate that the heterodimeric IDH $1^{\mathrm{R} / \mathrm{H}}$ would function in a way similar to the homodimeric IDH $1^{\mathrm{H} / \mathrm{H}}$, which accounts for the substantially reduced IDH activity of IDH $1^{\mathrm{R} / \mathrm{H}}$ at the physiological concentration of ICT and hence provides the mechanism underlying the observed dominant inhibitory effect of the R132H mutation [12]. Recently, Dang et al. [13] showed that the NADPH production is decreased when the wild-type and R132H mutant IDH1 are co-expressed and thus proposed that the mutant subunit might consume $\alpha \mathrm{KG}$ and NADPH produced locally by the wild-type subunit. Direct transfer of the produced $\alpha \mathrm{KG}$ and NADPH from the wild-type subunit to the mutant subunit seems unlikely because the two active sites are located on the opposite sides of the dimer and the dimeric interface is very tight and compact; instead, the reduction in NADPH production most likely results from the inhibited activity of the heterodimeric mutant.

\section{Mechanism of the gained reduction activity of the $\mathrm{R} 132 \mathrm{H}$ mutant}

Recently, Dang et al. [13] reported that the R132H mutation confers IDH1 a new function of catalyzing the conversion of $\alpha \mathrm{KG}$ to $2 \mathrm{HG}$, which might be important in the formation and progression of gliomas. Here, we show 
A

A R132H IDH1
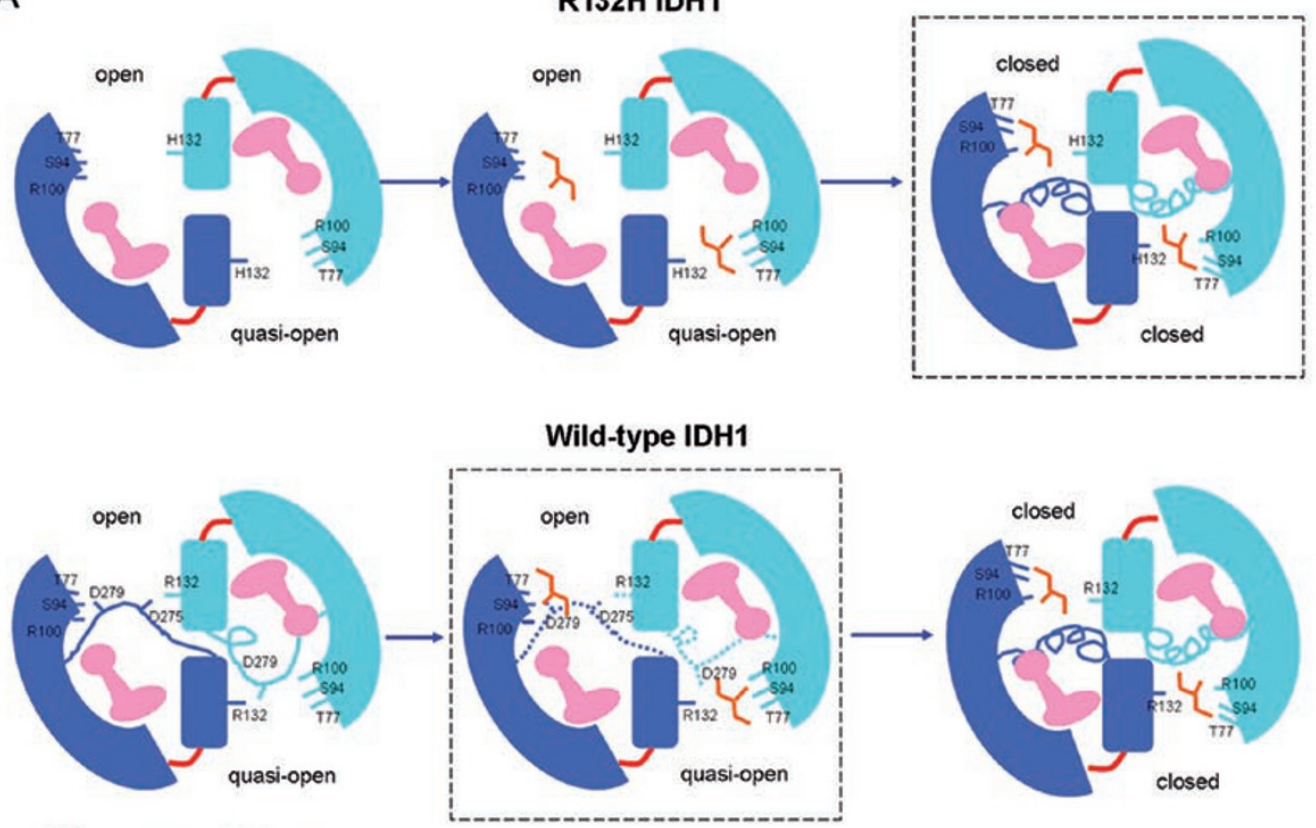

NADP $Y$ ICT

NADP-bound state

Initial ICT-binding state

Pre-transition state

B

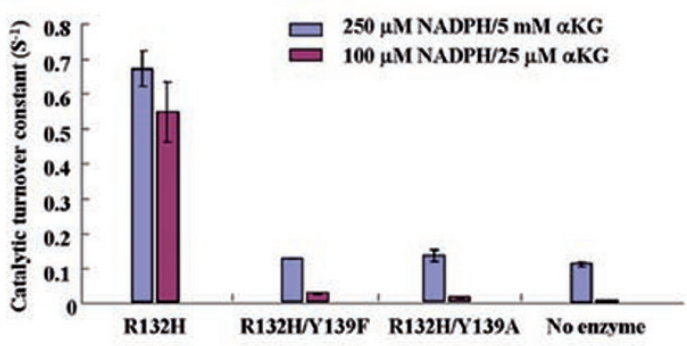

D

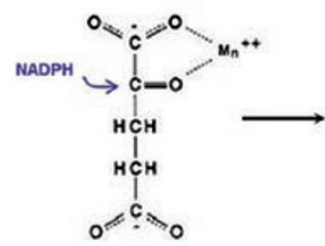

C
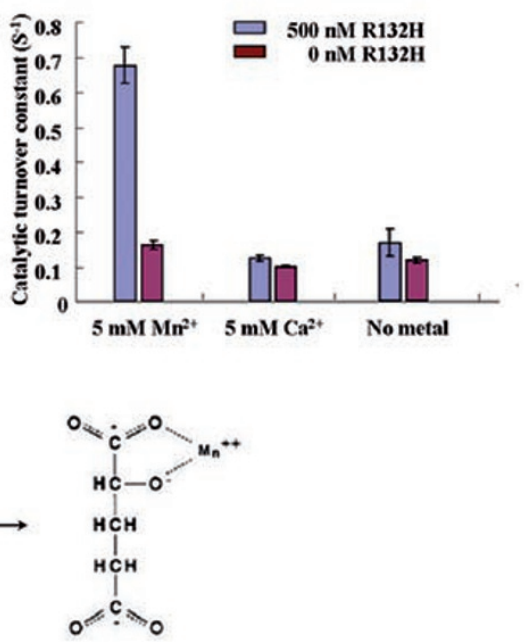

Figure 4 Mechanisms of "off-on switch" of the activities of IDH1 by the tumor-associated mutation R132H. (A) A schematic diagram illustrating the evolution of the active site of the wild-type and R132H mutant IDH1 from the NADP-bound state to the initial ICT-binding state and further to the pre-transition state in the ICT dehydrogenation reaction. Mutation of Arg132 to His enables the visualization of the inactive, initial ICT-binding state for the mutant IDH1. The transient initial ICT-binding state for the wild-type IDH1 and the high-energy pre-transition state for the R132H mutant IDH1 are not observed and thus are marked with dashed boxes. Residues Thr77, Thr99, and Arg100 that form the initial ICT-binding site, and residues Arg109 and Arg132 (or His132) of seg1 and Asp275 and Asp279 of seg2 that are involved in the assembly of the active site are labeled. (B) The $\alpha \mathrm{KG}$ reduction activities of the R132H, R132H/Y139F, and R132H/Y139A mutant IDH1. Negative controls with omission of the enzyme were performed to monitor the spontaneous turnover of $\alpha \mathrm{KG}$ to $2 \mathrm{HG}$ of the assay system. (C) Effects of metal ions on the reduction activity of the R132H mutant IDH1. Negative controls with omission of the enzyme and/or metal ion were included. (D) A schematic diagram illustrating the mechanism of the $\alpha \mathrm{KG}$ reduction to $2 \mathrm{HG}$ by the $\mathrm{R} 132 \mathrm{H}$ mutant $\mathrm{IDH} 1$. Tyr139 may play a critical role in the reduction of $\alpha \mathrm{KG}$ to $2 \mathrm{HG}$ by compensating the increased negative charge on the $\mathrm{C} 2$ atom of $\alpha \mathrm{KG}$ at the intermediate state. 
that the homodimeric R132H mutant harbours a weak reduction activity (Figure 4B) over a wide range of $\alpha \mathrm{KG}$ concentration $(10 \mu \mathrm{M}-1 \mathrm{mM}$; Supplementary information, Figure $\mathrm{S} 1 \mathrm{~A}$ ), and that $\mathrm{Mn}^{2+}$ but not $\mathrm{Ca}^{2+}$ facilitates the reaction (Figure $4 \mathrm{C}$ ). Spontaneous turnover of $\alpha \mathrm{KG}$ to $2 \mathrm{HG}$ is insignificant unless the $\alpha \mathrm{KG}$ concentration is very high (e.g., $5 \mathrm{mM}$; Figure 4B). The structure of the homodimeric R132H mutant in complex with NADP, $\alpha \mathrm{KG}$, and $\mathrm{Ca}^{2+}\left(\mathrm{IDH} 1^{\mathrm{H} / \mathrm{H}}-\mathrm{NADP}-\alpha \mathrm{KG}-\mathrm{Ca}\right)$ reported by Dang et al. [13] represents a post-transition state of the ICT dehydrogenation reaction and the pre-transition state of the $\alpha \mathrm{KG}$ reduction reaction. It has been noted that the IDH $1{ }^{\mathrm{H} / \mathrm{H}}-\mathrm{NADP}-\alpha \mathrm{KG}-\mathrm{Ca}$ structure [13] is very similar to the IDH1 $1^{\mathrm{R} / \mathrm{R}}$-NADP-ICT-Ca structure [14] except that Tyr139 displays a marked positional displacement [13]. This conformational difference should be rendered by the $\mathrm{R} 132 \mathrm{H}$ mutation only because the active sites of the quaternary complexes of ICT-bound IDH1 [14] and $\alpha \mathrm{KG}$-bound Idp1 [18] are very similar, while $\alpha \mathrm{KG}$ bound $\mathrm{IDH} 1^{\mathrm{H} / \mathrm{H}}[13]$ and Idp1 [18] differ mainly at this position (Supplementary information, Figure S1B). Our data prove the necessity of Tyr139 for the gained function by showing that the reduction activity of the R132H mutant is almost abolished when Tyr139 is substituted with either Phe or Ala (Figure 4B).

We further hypothesize that the reduction of $\alpha \mathrm{KG}$ is initiated by the transfer of a hydride anion from NADPH to the $\mathrm{C} 2$ atom of $\alpha \mathrm{KG}$, and Tyr139 may play a vital role by compensating the increased negative charge on the $\mathrm{C} 2$ atom at the intermediate state (Figure 4D). Detailed structural analyses show that the hydroxyl group of the displaced Tyr139 is at a distance of $3.7 \AA$ from the $\mathrm{C} 2$ atom of $\alpha \mathrm{KG}$ in the IDH $1^{\mathrm{H} / \mathrm{H}}$-NADP- $\alpha \mathrm{KG}-\mathrm{Ca}$ complex [13], while the corresponding distance is $5.8 \AA$ in the Idp1-NADP- $\alpha$ KG-Ca complex [18] which would be too far for a similar interaction. Therefore, appropriate positioning of the phenolic hydroxyl of Tyr139 would be required for its interaction with the $\mathrm{C} 2$ atom of $\alpha \mathrm{KG}$ and the reduction of the keto group of $\alpha \mathrm{KG}$, which is achieved in the R132H mutant. The metal ion may also be involved in the stabilization of the intermediate state by neutralizing the negative charge on the oxygen atom of the keto group (Figure 4D).

Taken together, our structural and biochemical data reveal a transient, initial ICT-binding state undiscovered before, implicate multiple functional roles of Arg132 in the catalytic reaction, and provide new insights into the catalytic mechanism of the ICT dehydrogenation reaction by IDH1. In particular, our results demonstrate that mutation of Arg 132 to His does not prohibit the ICT binding, instead, it causes the enzyme to be constrained in the inactive, initial ICT-binding state and prevents the assem- bly of the active, pre-transition state, providing in part the molecular basis for the impaired IDH activity of the R132H mutant. As the wild-type IDH1 may act as a tumor suppressor in part through suppression of the HIF $1 \alpha$ pathway by $\alpha \mathrm{KG}$ [12], our results provide insights into the mechanism of the "off switch" of the potential tumor suppressive function of IDH1 by the R132H mutation. Furthermore, our structural and biochemical data also show that mutation of Arg132 to His causes a positional displacement of Tyr139, which is optimal to be involved in the $\alpha K G$ reduction reaction. Since the reduction product $2 \mathrm{HG}$ is implicated to play a role in the pathogenesis of brain tumors [13], our results also provide insights into the mechanism of the "on switch" of the hypothetically oncogenic function of IDH1 by the R132H mutation. These findings may have important implications in diagnosis of cancers associated with the IDH1 mutations and in the design of therapeutic strategies for these cancers.

\section{Materials and Methods}

\section{Cloning, expression, and purification}

The pRSF-Duet-IDH1 $1^{\mathrm{R} 132 \mathrm{H}}$ plasmid was kindly provided by Dr Yue Xiong at Fudan University and used for expression of the homodimeric IDH1 $1^{\mathrm{H} / \mathrm{H}}$. Protein purification was carried out with Ni-NTA affinity chromatography and the target protein was eluted with an elution buffer (20 mM Tris- $\mathrm{HCl}$ (pH 7.4), $200 \mathrm{mM}$ imidazole, and $500 \mathrm{mM} \mathrm{NaCl}$ ). The protein was subsequently purified by gel filtration in a storage buffer $(20 \mathrm{mM}$ Tris- $\mathrm{HCl}(\mathrm{pH} 7.4)$ and $100 \mathrm{mM} \mathrm{NaCl}$ ) using a Hiload 16/60 Superdex G200 column (GE Healthcare).

The wild-type IDH1 was expressed with the pET-22b(+)IDH1 plasmid as described previously [14]. For expression of the heterodimeric $\mathrm{IDH} 1^{\mathrm{R} / \mathrm{H}}$, the gene fragment of the wild-type $I D H 1$ was cloned to pGEX-4T1, and E. coli BL21(DE3) cells were cotransformed with the pRSF-Duet-IDHI ${ }^{\mathrm{R} 132 \mathrm{H}}$ and pGEX-4T1-IDH1 plasmids.

Purification of the heterodimer was performed as described above except that the elution fractions from the Ni-NTA affinity chromatography were subjected to further purification using glutathione affinity column (GE Healthcare) and subsequent enzymatic digestion using thrombin (GE Healthcare), followed by final purification using gel filtration in a storage buffer $(20 \mathrm{mM}$ Tris- $\mathrm{HCl}(\mathrm{pH}$ 7.4) and $100 \mathrm{mM} \mathrm{NaCl}$ ) on a Hiload 16/60 Superdex G200 column (GE Healthcare). Further analysis of the purified protein with native-PAGE showed that it has a migration rate different from that of the homozygous proteins, confirming that it is the heterozygous enzyme. The constructs of the mutant IDH1 containing point mutations except for R132H were generated using the pET-22b-IDHI plasmid as the template with QuikChange Site-directed Mutagenesis kit (Stratagene). Expression and purification of these IDH1 mutants were carried out as described previously [14].

\section{Crystallization and diffraction data collection}

Crystallization conditions were screened using the Hampton 
Research Screen kits using the sitting-drop vapor diffusion method by Mosquito (TTP Labtech) at both $4{ }^{\circ} \mathrm{C}$ and $20{ }^{\circ} \mathrm{C}$. The crystals of the IDH $1^{\mathrm{H} / \mathrm{H}}$-NADP complex were grown in drops consisting of equal volumes $(0.5 \mu \mathrm{l})$ of the protein solution $\left(10 \mathrm{mg} \mathrm{ml}^{-1}\right)$ and the reservoir solution (1.0 $\mathrm{M} \mathrm{Na}_{2} \mathrm{HPO}_{4} / \mathrm{KH}_{2} \mathrm{PO}_{4}(\mathrm{pH}$ 8.2)) without the addition of NADP at $4{ }^{\circ} \mathrm{C}$. For crystallization screening of the IDH $1^{\mathrm{H} / \mathrm{H}}$ and IDH $1^{\mathrm{R} / \mathrm{H}}$ mutants in complexes with NADP and ICT, the protein solution was supplemented with $10 \mathrm{mM}$ NADP, $100 \mathrm{mM} \mathrm{ICT}$, and $100 \mathrm{mM} \mathrm{CaCl}_{2}$. The crystals of the IDH1H/HNADP-ICT complex were grown in drops containing the reservoir solution of $0.1 \mathrm{M}$ Tris- $\mathrm{HCl}(\mathrm{pH} 8.5)$ and $2.0 \mathrm{M}\left(\mathrm{NH}_{4}\right)_{2} \mathrm{SO}_{4}$, and that of the IDH $1^{\mathrm{R} / \mathrm{H}}$-NADP-ICT complex were obtained from drops containing the reservoir solution of 0.1 M HEPES-Na ( $\mathrm{pH} 7.5$ ), $2 \%$ PEG400, and $2.0 \mathrm{M}\left(\mathrm{NH}_{4}\right)_{2} \mathrm{SO}_{4}$. Diffraction data of the IDH $1^{\mathrm{H} /}$

${ }^{\mathrm{H}}$ binary complex were collected at beamline NW12 of Photon Factory, Japan, and that of the IDH $1^{\mathrm{H} / \mathrm{H}}$ and IDH $1^{\mathrm{R} / \mathrm{H}}$ ternary complexes at beamline BL17U1 of SSRF, China. All the diffraction datasets were processed using the HKL2000 program [28]. A summary of the diffraction data statistics is given in Table 1.

\section{Structure determination and refinement}

The crystal structures of the $\mathrm{R} 132 \mathrm{H}$ mutants were solved with the molecular replacement method implemented in the program PHASER of the CCP4 suite [29] using the structure of the IDH1 ${ }^{\mathrm{R} /}$ ${ }^{\mathrm{R}}$-NADP complex (PDB code 1T09) [14] as the search model. The initial structure refinement was carried out with the program $\mathrm{CNS}$ using the standard protocols [30] and the later structure refinement was performed with the programs REFMAC5 [31] and Phenix [32]. In addition, the IDH $1^{\mathrm{H} / \mathrm{H}}$-NADP complex was further refined with the newly released CNS v1.3. A free R factor monitor calculated with $5 \%$ randomly chosen reflections was applied throughout the refinement. In all three structures, there are two monomers in the asymmetric unit, which were refined independently. Model building was performed with the program COOT [33] and guided by SIGMAA-weighted $2 F_{O}-F_{C}$ and $F_{O}-F_{C}$ maps. The stereochemistry qualities of the structure models were analyzed using the program PROCHECK [34] and the structural analyses were carried out using programs of the CCP4 suite [29]. Figures were prepared using the program Pymol (http://www.pymol.org). A summary of the structure refinement statistics is given in Table 1 .

\section{IDH activity assay}

The IDH activities of both wild-type and mutant IDH1 were assayed spectrophotometrically by monitoring the reduction of NADP to NADPH, which has an extinction coefficient of 6220 $\mathrm{M}^{-1} \mathrm{~cm}^{-1}$ at $340 \mathrm{~nm}$. The standard reaction buffer $(1 \mathrm{ml})$ consisted of $30 \mathrm{mM}$ Tris-HCl (pH 7.4), $0.1 \mathrm{mM}$ NADP, $4 \mathrm{mM}$ DL-ICT, and $2 \mathrm{mM} \mathrm{MnSO}_{4}$ [25]. The reaction was initiated by adding $5 \mu \mathrm{l}$ of the wild-type or mutant IDH1 at appropriate concentrations. For determining the $K_{\mathrm{m}}$ of ICT, the concentration of ICT was varied, whereas NADP and $\mathrm{Mn}^{2+}$ were maintained at the standard concentrations. All kinetic data were measured using a Beckman DU800 spectrophotometer (Beckman Coulter) and analyzed using the program Prism 4.0 (Graphpad Software). The kinetic data were fitted to the Scatchard plot for apparent $K_{\mathrm{m}}$ and approximate $V_{\max }$ which was used to calculate the $k_{\text {cat }}$. All the experiments were performed in triplicates.

$\alpha K G$ reduction activity assay

The $\alpha \mathrm{KG}$ reduction activities of the R132H, R132H/Y139F, and R132H/Y139A IDH1 mutants were assayed by measuring the conversion of NADPH to NADP. Briefly, $500 \mathrm{nM}$ of the IDH1 mutant was added to $1 \mathrm{ml}$ of an assay solution consisting of $30 \mathrm{mM}$ Tris-Cl (pH 7.4), $5 \mathrm{mM} \mathrm{MnSO}_{4}, 250 \mu \mathrm{M} \beta-\mathrm{NADPH}$, and $5 \mathrm{mM}$ $\alpha \mathrm{KG}$, and the decrease of absorbance at $340 \mathrm{~nm}$ over time was measured. To assay the reduction activity under the physiological condition, $100 \mu \mathrm{M} \beta$-NADPH and $25 \mu \mathrm{M} \alpha \mathrm{KG}$ were used whereas $\mathrm{Mn}^{2+}$ was maintained at the standard concentration. All the experiments were performed in triplicates.

\section{ITC analysis}

Thermodynamic studies of both wild-type and mutant IDH1 were carried out by ITC experiments at $25^{\circ} \mathrm{C}$ using an ITC200 calorimeter (GE healthcare). The protein samples were dialyzed against an assay buffer (30 mM Tris- $\mathrm{HCl}$ (pH 7.4), $100 \mathrm{mM} \mathrm{NaCl}$, and 5\% glycerol), and DL-ICT was dissolved in the same buffer. For the wild-type IDH1, titrations were conducted with 20 injections of $1 \mathrm{mM}$ ICT, $1 \mu \mathrm{l}$ each, into $0.2 \mathrm{ml}$ of $0.044 \mathrm{mM}$ IDH1 at an interval of $100 \mathrm{~s}$. For the T77A, S94A, R100A, and R132H mutants, titrations were performed in a similar way except that 2 $\mu 1$ ICT was injected each time with an interval of $120 \mathrm{~s}$, and for the T77A, S94A, and R100A mutants, the concentrations of the protein $(0.075 \mathrm{mM})$ and ICT $(2 \mathrm{mM})$ were increased for higher heat release. The thermodynamic parameters were obtained by nonlinear least-squares fitting of the data using the Microcal ORIGIN software. Heats of the reactions were corrected for the effect of dilution.

\section{Protein data bank accession codes}

The structures of the IDH $1{ }^{\mathrm{H} / \mathrm{H}}-\mathrm{NADP}, \mathrm{IDH} 1^{\mathrm{H} / \mathrm{H}}-\mathrm{NADP}-\mathrm{ICT}$, and IDH $1^{\mathrm{R} / \mathrm{H}}$-NADP-ICT complexes have been deposited with the RCSB Protein Data Bank under accession codes 3MAR, 3MAP, and 3MAS, respectively.

\section{Acknowledgments}

We thank the staff members at Photon Factory of Japan and Shanghai Synchrotron Radiation Facility (SSRF) of China for technical supports in diffraction data collection, and Sheng Li, Jian $\mathrm{Wu}$, and Xianchi Dong of our group for technical assistance and helpful discussion. This work was supported by Grants from the Ministry of Science and Technology of China (2006AA02A313 and 2007CB914302), the National Natural Science Foundation of China (30730028), and the Chinese Academy of Sciences (KSCX2-YW-R-107 and SIBS2008002).

\section{References}

1 Balss J, Meyer J, Mueller W, Korshunov A, Hartmann C, von Deimling A. Analysis of the IDH1 codon 132 mutation in brain tumors. Acta Neuropathol 2008; 116:597-602.

2 Bleeker FE, Lamba S, Leenstra S, et al. IDH1 mutations at residue p.R132 $\left(I D H 1^{R 132}\right)$ occur frequently in high-grade gliomas but not in other solid tumors. Hum Mutat 2009; 30:711.

3 Yan H, Parsons DW, Jin G, et al. IDH1 and IDH2 mutations in gliomas. N Engl J Med 2009; 360:765-773.

4 Watanabe T, Nobusawa S, Kleihues P, Ohgaki H. IDH1 mutations are early events in the development of astrocytomas and oligodendrogliomas. Am J Pathol 2009; 174:1149-1153. 
5 Ichimura K, Pearson DM, Kocialkowski S, et al. IDH1 mutations are present in the majority of common adult gliomas but rare in primary glioblastomas. Neuro Oncol 2009; 11:341347.

6 Hartmann C, Meyer J, Balss J, et al. Type and frequency of IDH1 and IDH2 mutations are related to astrocytic and oligodendroglial differentiation and age: a study of 1,010 diffuse gliomas. Acta Neuropathol 2009; 118:469-474.

7 Hayden JT, Fruhwald MC, Hasselblatt M, Ellison DW, Bailey S, Clifford SC. Frequent IDH1 mutations in supratentorial primitive neuroectodermal tumors (sPNET) of adults but not children. Cell Cycle 2009; 8:1806-1807.

8 Verhaak RGW, Hoadley KA, Purdom E, et al. Intergrated genomic analysis identifies clinically relevant subtypes of glioblastoma characterized by abnormalities in PDGFRA, IDH1, EGFR, and NF1. Cancer Cell 2010; 17:98-110.

9 Mardis ER, Ding L, Dooling DJ, et al. Recurring mutations found by sequencing an acute myeloid leukemia genome. $N$ Engl J Med 2009; 361:1058-1066.

10 Kang MR, Kim MS, Oh JE, et al. Mutational analysis of IDH1 codon 132 in glioblastomas and other common cancers. Int $J$ Cancer 2009; 125:353-355.

11 Sjoblom T, Jones S, Wood LD, et al. The consensus coding sequences of human breast and colorectal cancers. Science 2006; 314:268-274.

12 Zhao S, Lin Y, Xu W, et al. Glioma-derived mutations in IDH1 dominantly inhibit IDH1 catalytic activity and induce HIF-1a. Science 2009; 324:261-265.

13 Dang L, White DW, Gross S, et al. Cancer-associated IDH1 mutations produce 2-hydroxyglutarate. Nature 2009; 462:739744.

$14 \mathrm{Xu} \mathrm{X,} \mathrm{Zhao} \mathrm{J,} \mathrm{Xu} \mathrm{Z,} \mathrm{et} \mathrm{al.} \mathrm{Structures} \mathrm{of} \mathrm{human} \mathrm{cytosolic}$ NADP-dependent isocitrate dehydrogenase reveal a novel self-regulatory mechanism of activity. J Biol Chem 2004; 279:33946-33957.

15 Nekrutenko A, Hillis DM, Patton JC, Bradley RD, Baker RJ. Cytosolic isocitrate dehydrogenase in humans, mice, and voles and phylogenetic analysis of the enzyme family. Mol Biol Evol 1998; 15:1674-1684.

16 Gimelbrant A, Hutchinson JN, Thompson BR, Chess A. Widespread monoallelic expression on human autosomes. Science 2007; 318:1136-1140.

17 Ceccarelli C, Grodsky NB, Ariyaratne N, Colman RF, Bahnson BJ. Crystal structure of porcine mitochondrial $\mathrm{NADP}^{+}$dependent isocitrate dehydrogenase complexed with $\mathrm{Mn}^{2+}$ and isocitrate. Insights into the enzyme mechanism. $J$ Biol Chem 2002; 277:43454-43462.

18 Peng Y, Zhong C, Huang W, Ding J. Structural studies of Saccharomyces cerevesiae mitochondrial NADP-dependent isocitrate dehydrogenase in different enzymatic states reveal substantial conformational changes during the catalytic reaction. Protein Sci 2008; 17:1542-1554.

19 Hurley JH, Thorsness PE, Ramalingam V, Helmers NH, Koshland DE, Jr., Stroud RM. Structure of a bacterial enzyme regulated by phosphorylation, isocitrate dehydrogenase. Proc
Natl Acad Sci USA 1989; 86:8635-8639.

20 Yasutake Y, Watanabe S, Yao M, Takada Y, Fukunaga N, Tanaka I. Structure of the monomeric isocitrate dehydrogenase: evidence of a protein monomerization by a domain duplication. Structure 2002; 10:1637-1648.

21 Karlstrom M, Stokke R, Steen IH, Birkeland NK, Ladenstein $R$. Isocitrate dehydrogenase from the hyperthermophile Aeropyrum pernix: X-ray structure analysis of a ternary enzymesubstrate complex and thermal stability. J Mol Biol 2005; 345:559-577.

22 Kim TK, Lee P, Colman RF. Critical role of Lys212 and Tyr140 in porcine NADP-dependent isocitrate dehydrogenase. J Biol Chem 2003; 278:49323-49331.

23 Dean AM, Koshland DE, Jr. Kinetic mechanism of Escherichia coli isocitrate dehydrogenase. Biochemistry 1993; 32:9302-9309.

24 Soundar S, Danek BL, Colman RF. Identification by mutagenesis of arginines in the substrate binding site of the porcine NADP-dependent isocitrate dehydrogenase. $J$ Biol Chem 2000; 275:5606-5612.

25 Grodsky NB, Soundar S, Colman RF. Evaluation by sitedirected mutagenesis of aspartic acid residues in the metal site of pig heart NADP-dependent isocitrate dehydrogenase. Biochemistry 2000; 39:2193-2200.

26 Kim TK, Colman RF. Ser95, Asn97, and Thr78 are important for the catalytic function of porcine NADP-dependent isocitrate dehydrogenase. Protein Sci 2005; 14:140-147.

27 Lee ME, Dyer DH, Klein OD, Bolduc JM, Stoddard BL, Koshland DE Jr. Mutational analysis of the catalytic residues lysine 230 and tyrosine 160 in the $\mathrm{NADP}^{+}$-dependent isocitrate dehydrogenase from Escherichia coli. Biochemistry 1995; 34:378-384.

28 Otwinowski Z, Minor W. Processing of X-ray diffraction data collected in oscillation mode. Methods in Enzymology 1997; 276:307-326.

29 Collaborative Computational Project, Number 4. The CCP4 suite: programs for protein crystallography. Acta Crystallogr D Biol Crystallogr 1994; 50(Pt 5):760-763.

30 Brunger AT, Adams PD, Clore GM, et al. Crystallography \& NMR system: a new software suite for macromolecular structure determination. Acta Crystallogr D Biol Crystallogr 1998; 54(Pt 5):905-921.

31 Murshudov GN, Vagin AA, Dodson EJ. Refinement of macromolecular structures by the maximum-likelihood method. Acta Crystallogr D Biol Crystallogr 1997; 53(Pt 3):240-255.

32 Adams PD, Afonine PV, Bunkoczi G, et al. PHENIX: a comprehensive Python-based system for macromolecular structure solution. Acta Crystallogr D Biol Crystallogr 2010; 66(Pt 2):213-221.

33 Emsley P, Cowtan K. Coot: model-building tools for molecular graphics. Acta Crystallogr D Biol Crystallogr 2004; 60(Pt 12 Pt 1):2126-2132.

34 Laskowski RA, Moss DS, Thornton JM. Main-chain bond lengths and bond angles in protein structures. $J \mathrm{Mol} \mathrm{Biol}$ 1993; 231:1049-1067.

(Supplementary information is linked to the online version of the paper on the Cell Research website.) 